\title{
Greetings from the New SIGCHI Leadership
}

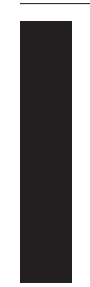

am very pleased to write my first message as president of SIGCHI, and to introduce myself and the new executive vice president, Helena Mentis. As of this writing, the rest of the new SIGCHI

Executive Committee has not yet been finalized; we'll introduce them in later messages.

I am a professor in the Department of Computer Science and Engineering at the University of Minnesota. I've been here since 2002, and before that I spent 11 years at Bell Labs and AT\&T Labs. My focus areas are social computing and crowdsourcing. I've served the SIGCHI community in a number of ways, including co-chairing CHI 2002, serving as program co-chair for CSCW twice, co-chairing IUI 1998, and serving on the SIGCHI Executive Committee for the past six years.

Helena is an assistant professor of information systems at the University of Maryland, Baltimore County. Her focus areas are CSCW and health informatics. She has been a part of the SIGCHI community since 2002, when she attended her first CHI conference in snowy Minneapolis. Since then she has started three local SIGCHI chapters, been a conference committee member for CHI and CSCW, and served as an adjunct chair for four different SIGCHI conferences.

During our three-year term, we intend to focus on the following issues:

Outreach and publicity. Building on SIGCHI's success, we want to promote participation at all levels by groups traditionally underrepresented in computing. We seek to evangelize our record and success to the rest of ACM and the broader computing community. The SIGCHI community has much to offer other fields of practice and research; thus, we will explore getting SIGCHI-related publications included in appropriate scholarly databases.

Modernizing our processes.

Participation in our flagship conferences has increased dramatically; therefore, we must update our processes to make sure they scale. We also must examine our reviewing and publication processes to be sure they serve community goals. We will address needs through a combination of policy, technology, and well-defined mentorship.

Internationalization. SIGCHI has worked to move beyond our traditional base of North America and Europe. We will continue this effort, evaluating the success of past endeavors as a step toward determining future steps.
Openness. We will work to increase communication between SIGCHI leadership and members. We will use modern communication media effectively to open community deliberations, discussions, and data to the greatest possible extent. We will also work to engage new and younger members of our community in service at all levels.

But at this stage, it's important that we hear from you, the SIGCHI membership and the broader HCI community. What do you think of the state and future of our field? Get in on the ground floor, share your ideas, and join our conversation. There are lots of ways to contact us and interact with the HCI community: For starters, you can email us at sigchi-president@ acm.org and sigchi-executive-sp@acm. org, tweet to us at @sig_chi, and join the ACM SIGCHI Facebook group. And don't forget to check out SIGCHI communities: http://www.sigchi. org/communities. Here you can get together with others who share your interests to make sure your voice is heard within SIGCHI.

(1) Loren Terveen, President, ACM SIGCHI $\rightarrow$ terveenacs.umn.edu

\section{We want to promote participation at all levels by groups traditionally underrepresented in computing.}

